rule. However, some exceptions have been recently reported. For example, from a study of the wavelength dependence of the intensity of benzene-sensitized fluorescence of $p$-terphenyl, Braun, Kato, and Lipsky ${ }^{4}$

4. Lipsky, C. L. Braun, and S. Kato, J. Chem. Phys. 37, 190 (1962). Additional references in this paper. concluded that this rule breaks down in the case of benzene and its alkyl derivatives, suggesting that the higher excited states of the donor are not converted quantitatively into the lowest excited state.

A detailed discussion of these results will be presented elsewhere.

\title{
Separation of Sets of Variables in Quantum Mechanics*
}

\author{
R. A. Marcus $\dagger$ \\ Department of Chemistry, Brookhaven National Laboratory, Upton, New York and \\ Department of Chemistry, Polytechnic Institute of Brooklyn, Brooklyn, New York
}

(Received 5 March 1964)

\begin{abstract}
Separation of the Schrödinger equation for molecular dynamics into sets of variables can sometimes be performed when separation into individual variables is neither possible nor for certain purposes necesary. Sufficient conditions for such a separation are derived. They are the same as those found by Stäckel for the corresponding Hamilton-Jacobi problem, with an additional one which is the analog of the Robertson condition for one-dimensional sets.

Expressions are also derived for operators whose eigenvalues are the separation constants. They provide a variational property for these constants. For use in aperiodic problems an expression is obtained for the probability current in curvilinear coordinates in an invariant form. Application of these results to reaction rate theory is made elsewhere.
\end{abstract}

\section{INTRODUCTION}

$\mathbf{I}^{\mathrm{N}}$ N 1891, Stäckel obtained the necessary and sufficient conditions for separation of variables in the Hamilton-Jacobi equation, separating a partial differential equation for a system of $n$ degrees of freedom into $n$ ordinary differential equations. ${ }^{1}$ Later, Robertson extended these results to the $n$-dimensional Schrödinger equation, obtaining conditions the same as those found by Stäckel, plus one additional one. ${ }^{2}$ The differential geometric implication of this last condition has been described by Eisenhart. ${ }^{3}$

In 1897, Stäckel generalized his earlier work, by considering the conditions for separation of the Hamilton-Jacobi equation into sets of variables. ${ }^{4}$ His results

\footnotetext{
* Research performed in part under auspices of the U.S. Atomic Energy Commission.

+ Visiting Senior Scientist, B.N.L. Present address: Noyes Chemical Laboratory, University of Illinois, Urbana, Illinois. This research was supported in part by a fellowship from the Alfred P. Sloan Foundation.

1 P. Stäckel, Habilitationschift, Halle (1891). (I am indebted to the librarian of Princeton University for a photocopy of this manuscript.) In deriving the necessary conditions Stäckel considered a general coordinate system; in deriving the sufficient ones he considered orthogonal systems.

${ }^{2}$ H. P. Robertson, Math. Ann. 98, 749 (1927). The similarity of conditions noted refers to those for the only coordinate systems considered by Robertson, namely, orthogonal ones. Parenthetically it may be noted that in some non-Euclidean spaces for which $n>3$, it is impossible to find a set of orthogonal coordinates [e.g. Ref. 7 (b) pp. 45 and 104].

${ }^{3}$ L. P. Eisenhart, Ann. Math. 35, 284 (1934)

4P. Stäckel, Ann. Mat. Pura Appl. Ser. 2A 25, 55 (1897).
}

reduced to those obtained previously ${ }^{1}$ when each set consisted of only one coordinate. In the present paper, this result is extended to the $n$-dimensional Schrödinger equation, using arguments paralleling those employed by Robertson and Stäckel. An application to reaction rate theory is given elsewhere. ${ }^{5}$

In a subsequent section an expression for the probability current in curvilinear coordinates is derived by using the standard expression for the gradient in tensor calculus. (No separation of variables is assumed.) The expression has application to aperiodic problems. In a concluding section of the paper operators are formed whose eigenvalues are the separation constants. A variational property of these constants is then derived for possible use in approximations.

\section{DERIVATION OF SOME CONDITIONS FOR SEPARATION}

The $n$-dimensional Schrödinger equation has the form ${ }^{6}$

$$
\sum_{r, s=1}^{n}(g)-\frac{\partial}{\partial q_{r}}\left(g^{t} g^{r s} \frac{\partial \psi}{\partial q_{s}}\right)+k^{2}\left(\alpha_{1}-V\right) \psi=0,
$$

where $k^{2}$ equals $2 / \hbar^{2}, \alpha_{1}$ is the energy $E, V$ is the potential energy, and $\psi$ the wavefunction for the entire system. $g^{r s}$ is reciprocal to $g_{r s}$. The latter appear in the

\footnotetext{
- R. A. Marcus (to be published).

B E.g., W. Pauli, Jr., Handbuch der Physik, edited by S. Flugge (Springer-Verlag, Berlin, 1958), Vol. 5, p. 39.
} 
fundamental line element $d s$ in a mass-weighted space having the $q^{r}$ as generalized coordinates and are defined in $(2 \mathrm{~b})$

$$
d s^{2}=\sum_{r, s=1}^{n} g_{r s} d q^{r} d q^{s}
$$

If the coordinates $q^{r}$ are the ordinary Cartesian coordinates $x^{r}$, then $g_{r s}$ equals $m_{r} \delta_{r s}$, where $m_{r}$ is the mass of the atom whose coordinates include this $x^{r}$ and where $\delta_{r s}$ is the Kronecker delta function. If the coordinates $q^{r}$ are any other ones, the corresponding $g_{r s}$ and $g^{r s}$ are then computed from the expressions

$$
g_{r_{s}}=\sum_{i=1}^{n} m_{i} \frac{\partial x^{i}}{\partial q^{r}} \frac{\partial x^{i}}{\partial q^{s}}, \quad g^{r s}=\sum_{i=1}^{n} \frac{1}{m_{i}} \frac{\partial q^{r}}{\partial x^{i}} \frac{\partial q^{s}}{\partial x^{i}} .
$$

For example, if the $q^{r}$ are mass-weighted Cartesian coordinates, i.e., if they equal $\left(m_{r}\right)^{\frac{1}{3}} x^{r}$, then $g_{r s}$ equals $\delta_{r s}$.

Robertson considered systems having orthogonal coordinates $\left(g^{r s}=0\right.$ if $\left.r \neq s\right){ }^{7}$ In generalizing to sets of coordinates, we consider (as did Stäckel in 1897) systems for which any coordinates belonging to different sets are orthogonal, ${ }^{8}$ and so those for which $g^{r s}$ vanishes when $r$ and $s$ belong to different sets.

The $\mu$ th set is denoted by an index $\mu$. Let it contain $h_{\mu}$ coordinates and let there be $m$ sets. Then,

$$
\sum_{\mu=1}^{m} h_{\mu}=n \text {. }
$$

The coordinates in the $\mu$ th set are denoted by $q^{\mu i}$ $\left(i=1\right.$ to $\left.h_{\mu}\right)$ and will be referred to as set $\mu$. The Schrödinger equation (1) then becomes

$$
(g)^{-\frac{1}{2}} \sum_{\mu=1}^{m} \sum_{i, j=1}^{h_{\mu}} \frac{\partial}{\partial q^{\mu_{i}}} g^{\frac{1}{2}} g^{\mu_{i} \mu_{j}} \frac{\partial \psi}{\partial q^{\mu_{i}}}+k^{2}\left(\alpha_{1}-V\right) \psi=0 .
$$

To find some conditions for the separation, we shall impose Condition (3) on the $g^{\mu_{i} \mu_{j}}$. Eventually conditions will be found in which (3) plays a role. They will be shown later to be sufficient to effect separation rather than to be necessary:

$$
g^{\mu i \mu_{i}} g^{\frac{1}{2}}=f^{\prime \mu_{i \mu j}} a_{\mu},
$$

where $f^{\prime \mu_{i \mu j}}$ depends only on set $\mu$ and where $a_{\mu}$ depends only on coordinates in the remaining $m-1$ sets. Upon introduction of (3) and (4), where $\psi_{\mu}$ depends only on Set $\mu,(2)$ yields (5)

$$
\psi=\prod_{\mu=1}^{m} \psi_{\mu *}
$$

\footnotetext{
${ }^{7}$ (a) More precisely, when the coordinates are orthogonal the quantities reciprocal to $g^{r s}, g_{r s}$, are diagonal. However, since $g g^{r a}$ is the cofactor of $g_{r s}$, where $g$ is the $n \times n$ determinant of the $g_{r a}$, and since $g_{r s}$ is diagonal, so is $g^{r}$. For example, (b) C. E. Weatherburn, Riemannian Geometry (Cambridge University Press, New York, 1957), Eq. (14), p. 41.

8 In this case, by suitable numbering of the coordinates, the nonvanishing elements in the $g_{r}$ determinant occur in blocks along the diagonal, one block for each set of coordinates; $g_{r a}$ vanishes when $r$ and $s$ belong to different sets. It can then be shown that $g^{r s}$ also vanishes for such pairs of $r$ and $s$.
}

(Problems in which there are symmetry or antisymmetry conditions on $q^{r \prime}$ s drawn from different $\mu$ sets are not considered here.)

$$
(g)^{-1} \sum_{\mu} \frac{a_{\mu}}{\psi_{\mu}} \sum_{i, j=1}^{k_{\mu}} \frac{\partial}{\partial q_{i}^{\mu_{i}}} f^{\prime_{\mu i \mu j}} \frac{\partial}{\partial q^{\mu_{i}}} \psi_{\mu}+k^{2}\left(\alpha_{1}-V\right)=0
$$

If Eq. (5) is separated into $m$ partial differential equations, one for each set $\psi_{\mu}$, there will be $m-1$ separation constants, $\alpha_{2}$ to $\alpha_{m}$. Each $\psi_{k}$ will depend on $\alpha_{1}$ to $\alpha_{m}$, and so (5) can be regarded as an identity in $\alpha_{1}$ to $\alpha_{m}$, i.e., it is satisfied for arbitrary values of $\alpha_{1}, \cdots, \alpha_{m}$ (when no boundary conditions are imposed). It can be differentiated with respect to any of these $\alpha_{\nu}$, noting that $V$ is of course independent of the $\alpha$ 's. One so obtains $m$ equations

$$
(g)^{-1} \sum_{\mu} a_{\mu} W_{\mu \nu}=\delta_{1},
$$

where $W_{\mu \nu}$ is a function only of the coordinates in the $\mu$ th set and of the $\alpha_{y}$ 's:

$$
W_{\mu \nu}=-\frac{1}{k^{2}} \frac{\partial}{\partial \alpha_{\nu}} \sum_{i, j=1}^{h_{\mu}} \frac{1}{\psi_{\mu}} \frac{\partial}{\partial q^{\mu_{i}}} f^{\prime \mu_{i j}} \frac{\partial \psi_{\mu}}{\partial q^{\mu_{i}}} .
$$

It must be possible to find a value of $\left(\alpha_{1}, \cdots, \alpha_{m}\right)$, say $\left(\alpha_{1}^{0}, \cdots, \alpha_{m}{ }^{0}\right)$, such that $\operatorname{det} W_{\mu \nu}$ in Eq. (7) does not vanish. (Otherwise, $\psi$ would depend on less than $m$ constants $\alpha_{p}$. Compare Appendix I.) Let the corresponding value of $W_{\mu \nu}$ be denoted by $\phi_{\mu \nu}{ }^{\prime}$. It depends no longer on $\left(\alpha_{1}, \cdots, \alpha_{n}\right)$. For this $\alpha=\alpha^{0}$, the functions conjugate to these $\phi_{\mu \nu}{ }^{\prime}, \phi^{\prime \mu \nu}$ exist since the determinant of the $\phi_{\mu \nu}{ }^{\prime}, \phi^{\prime}$, is nonzero. We may write

$$
\sum_{\mu=1}^{m} \phi^{\prime \mu v} \phi_{\mu \lambda}{ }^{\prime}=\delta_{\nu \lambda} .
$$

Comparison with (6) then shows that

$$
\phi^{\prime \mu 1}=a_{\mu} / g^{\frac{1}{2}} .
$$

According to (8) $\phi^{\prime} \phi^{\prime \mu \nu}$ is the cofactor of $\phi_{\mu \nu}{ }^{\prime}$ in $\phi^{\prime}$. Since each $\phi_{\mu \nu}{ }^{\prime}$ depends only on the coordinates in the $\mu$ th row, the cofactor $\phi^{\prime} \phi^{\prime \mu \nu}$ must be independent of them, as is $a_{\mu}$ by definition. From (9), it then follows that $\phi^{\prime} / g^{\frac{z}{3}}$ is independent of the coordinates of the $\mu$ th set. Since this independence holds for all $\mu$ we have

$$
\phi^{\prime} / g^{\frac{1}{2}}=K, \text { a constant. }
$$

From (3) and (9) we also have

$$
g^{\mu_{i \mu i}=} \phi^{\prime \mu 1} f^{\prime \mu_{i \mu} i} \text {. }
$$

Finally, Eq. (5) can be written as in (10), using (8) and (9):

$$
V=\sum_{\mu=1}^{m} \phi^{\prime \mu 1}\left(\alpha_{1} \phi_{\mu 1}{ }^{\prime}+\frac{1}{k^{2}} \frac{1}{\psi_{\mu}} \sum_{i, j=1}^{h_{\mu}} \frac{\partial}{\partial q^{\mu_{i}}} f^{\mu_{i \mu} \mu_{j}} \frac{\partial \psi_{\mu}}{\partial q^{\mu_{j}}}\right) .
$$

The rhs of (10) is only apparently a function of the $\alpha$ 's. Like $V$, it must actually be independent of them, and so is unchanged when the $\left(\alpha_{1}, \cdots, \alpha_{n}\right)$ is replaced by 
$\left(\alpha_{1}^{0}, \cdots, \alpha_{n}^{0}\right)$. The corresponding function in parentheses is a function of set $\mu$ alone and will be denoted by $X_{\mu}{ }^{\prime}$. Thus, a third condition for the separation of variables is

$$
V=\sum_{\mu} \phi^{\prime \mu 1} X_{\mu}{ }^{\prime}
$$

\section{CONDITIONS (I') TO (III') ARE SUFFICIENT}

Conditions $\left(\mathrm{I}^{\prime}\right)$ to $\left(\mathrm{III}^{\prime}\right)$ are sufficient for separation of the variables into the $m$ sets. The proof is as follows.

Inserting these conditions into the Schrödinger equation (2), noting that $\phi^{\prime} \phi^{\prime \mu 1}$ is independent of Set $\mu$, and using (8) one obtains

$$
\sum_{\mu} \phi^{\prime \mu 1} Y_{\mu}=0
$$

where

$$
Y_{\mu}=\frac{1}{\psi_{\mu}} \sum_{i, j=1}^{h_{\mu}} \frac{\partial}{\partial q^{\mu_{i}}} f^{\prime \mu_{i \mu}} \frac{\partial \psi_{\mu}}{\partial q^{\mu_{j}}}+k^{2}\left(\alpha_{1} \phi_{\mu 1}{ }^{\prime}-X_{\mu}{ }^{\prime}\right) .
$$

Consider any specified value of all the coordinates in the system. $\left(Y_{1}, \cdots, Y_{m}\right)$ can then be regarded as a vector in an $m$-dimensional space, with components $Y_{1}, \cdots, Y_{m}$. This vector is orthogonal to another vector $\left(\phi^{\prime 11}, \cdots, \phi^{\prime m 1}\right)$ in this same space. The subspace orthogonal to any vector $\left(\phi^{\prime 11}, \cdots, \phi^{m 1}\right)$ is $m-1$ dimensional. However, there are already $m-1$ linearly independent vectors orthogonal to $\left(\phi^{\prime 11}, \cdots, \phi^{\prime m 1}\right)$, namely $\left(\phi_{1 \lambda}{ }^{\prime}, \cdots, \phi_{m \lambda}{ }^{\prime}\right)$, where $\lambda=2$ to $m$ [cf. Eq. (8)]. It follows that $\left(Y_{1}, \cdots, Y_{m}\right)$ must be a linear combination of them. That is

$$
Y_{\mu}=-k^{2} \sum_{\nu=2}^{m} \alpha_{\nu} \phi_{\mu \nu}^{\prime},
$$

where the $\alpha_{\nu}$ are constants. Equation (13) holds for any set of specified values of all the coordinates, and hence over all configuration space.

Equations (12) and (13) yield the separated equations:

$$
\sum_{i, j=1}^{h \mu} \frac{\partial}{\partial q^{\mu_{i}}} f^{\prime \mu_{i} \mu_{j}} \frac{\partial \psi_{\mu}}{\partial q^{\mu_{j}}}+k^{2}\left(\sum_{\nu=1}^{m} \alpha_{\nu} \phi_{\mu \nu}{ }^{\prime}-X_{\mu}{ }^{\prime}\right) \psi_{\mu}=0 .
$$

In summary, one sees that given $m^{2}$ quantities, $\phi_{\mu \nu}{ }^{\prime}$ such that each is a function only of the set of variables described by the first index and such that the product of their determinant with $1 / g^{\frac{1}{2}}$ is a constant, then a separation of variables into sets of variables can be effected when conditions $\left(\mathrm{II}^{\prime}\right)$ and $\left(\mathrm{III}^{\prime}\right)$ are also fulfilled.

\section{COMPARISON WITH STÄCKEL AND ROBERTSON}

Stäckel found that Conditions ( $\left(\mathrm{II}^{\prime}\right)$ and $\left(\mathrm{III}^{\prime}\right)$ alone sufficed for separation of the Hamilton-Jacobi equation. $\left(\mathrm{I}^{\prime}\right)$ is the analog of the additional one found by Robertson for the case where each subspace $\mu$ is one dimensional.

For comparison with Robertson's results and for application it is convenient to define new quantities $\phi_{\mu \nu}, f^{\mu i \mu_{j}}, X_{\mu}$ and $\phi^{\mu \nu}$ :

$$
\begin{aligned}
\phi_{\mu \nu}^{\prime} & =\phi_{\mu \nu}{ }^{\prime} / f_{\mu}{ }^{\prime}, \quad f^{\mu i \mu j}=f^{\prime \mu_{i \mu j}} / K^{1 / m}, \\
X_{\mu} & =X_{\mu}{ }^{\prime} / f_{\mu}{ }^{\prime}, \quad \phi^{\mu \nu}=\phi^{\prime \mu \nu} f_{\mu}{ }^{\prime},
\end{aligned}
$$

where $f_{\mu}{ }^{\prime}$ is the $h_{\mu}$ th root of an $h_{\mu} \times h_{\mu}$ determinant,

$$
\operatorname{det}_{i, j=1}^{h_{\mu}} f^{\mu_{i} \mu_{j}}
$$

From (15) $\phi$ equals

$$
\phi^{\prime} / \prod_{\mu} f_{\mu}{ }^{\prime}
$$

We introduce $f_{\mu}$ :

$$
f_{\mu}=\left(\underset{i, j=1}{\operatorname{det}_{\mu}} f^{\mu_{i} \mu_{j}}\right)^{1 / h_{\mu}},
$$

which equals $f_{\mu}{ }^{\prime} / K^{1 / m}$. The quantities $f^{\mu i \mu i}, f_{\mu}$, and $\phi_{\mu \nu}$ are again functions only of the $\mu$ th set of variables. From (8) and (15) we also have

$$
\sum_{\mu=1}^{h_{\mu}} \phi^{\mu v} \phi_{\mu \lambda}=\delta_{\nu \lambda} .
$$

With these quantities, Conditions $\left(\mathrm{I}^{\prime}\right)$ to $\left(\mathrm{III}^{\prime}\right)$ become $^{9}$ the following conditions on the $g^{r s}$ and on $V$ :

$$
\begin{gathered}
\phi / g^{\frac{1}{2}}=1 / \prod_{\mu=1}^{m} f_{\mu}, \\
\left(\operatorname{det}_{i, j=1}^{h_{\mu}} g^{\mu_{i} \mu_{j}}\right)^{1 / h_{\mu}}=\phi^{\mu 1}, \\
V=\sum_{\mu=1}^{m} \phi^{\mu 1} X_{\mu .}
\end{gathered}
$$

The separated equations become

$$
\frac{1}{f_{\mu}} \sum_{i, j=1}^{h_{\mu}} \frac{\partial}{\partial q^{\mu}} f^{\mu_{i \mu} \mu_{j}} \frac{\partial}{\partial q^{\mu_{i}}} \psi_{\mu}+k^{2}\left(\sum_{\nu=1}^{m} \alpha_{\nu} \phi_{\mu \nu}-X_{\mu}\right) \psi_{\mu}=0
$$

where

$$
f^{\mu_{i \mu j}}=g^{\mu_{i} \mu_{j}} f_{\mu} /\left(\operatorname{det}_{i, j=1}^{h_{\mu}} g^{\mu_{i} \mu_{j}}\right)^{1 / h_{\mu}}
$$

In the special case that all the subspaces $\mu$ are onedimensional there is only one $f^{\mu_{i} \mu_{j}}$ for each $\mu$, and so the latter equals its determinant $f_{\mu}$. There is also only one $g^{\mu i \mu_{j}}$ for each $\mu$, which then equals its determinant. The conditions and the separated equations then reduce to those obtained by Robertson.

Given a set of coordinates which permit a separation into sets of variables and given some method (not specified) for determining the Stäckel coefficients $\phi_{\mu \nu}$, Eq. (I) provides the values for the $f_{\mu}$ 's: Each $f_{\mu}$ is the factor in $\phi / g^{\frac{1}{2}}$ which depends only on set $\mu$. Equa-

${ }^{9}$ To obtain (II) one first computes a determinant from (II'):

$$
\operatorname{det}_{i, j=1}^{h_{\mu}} g^{\mu_{i} \mu_{j}}=\left(\phi^{\prime \mu 1}\right)^{h_{\mu}} \operatorname{det}_{i, j=1}^{h_{\mu}} f^{\mu_{i} \mu_{i}}
$$


tion (18) is then used to determine the $f^{\mu i \mu j^{\prime}}$ 's. The separated equations are given by (17).

The problems of determining the systems of subspaces which permit separation of variables and of determining the Stäckel coefficients remain as separate problems. Eisenhart solved the one-dimensional analogs of Conditions (I) to (III) and determined thereby all the coordinate systems in $n$ dimensions which permit separation of the Schrödinger equation into $n$ onedimensional equations. ${ }^{3} \mathrm{He}$ has tabulated the Stäckel coefficients for $n=3$ but not for larger $n$. Presumably those for larger $n$ can be derived from various results in his paper. ${ }^{10}$ Then again, a study analogous to Eisenhart's but devised for several-dimensional sets rather than for purely one-dimensional ones would be of interest here.

To illustrate for later use how a common case falls within the formalism embodied in Eqs. (I) to (III), (17) and (18), we consider in Appendix II the separation of rotation from vibration in a diatomic molecule.

\section{PROBABILITY CURRENT IN CURVILINEAR COORDINATES}

Usually, literature expressions for the probability current across a surface are given for Cartesian or spherical polar coordinates, sometimes mass weighted, more often not. ${ }^{11}$ By using an expression for the current in an invariant form and then introducing the standard form for $\boldsymbol{\nabla}$ in Riemannian geometry one may obtain the current in curvilinear coordinates. The result is given by Eq. (23). It reduces to (24) when the surface being crossed is a coordinate hypersurface. The result is then used to obtain an expression for the transmission coefficient.

It will be recalled ${ }^{12}$ that from the time-dependent Schrödinger equation, $H \psi=(\hbar / i) \partial \psi / \partial t$, an equation of continuity (19) can be derived, where the probability density $\rho$ is $\psi^{*} \psi$ and where $I$ is a vector defined by (20):

$$
\begin{gathered}
(\partial \rho / \partial t)+\nabla \cdot \mathbf{I}=0, \\
\mathbf{I}=(\hbar / 2 i)\left(\psi^{*} \nabla \psi-\psi \nabla \psi^{*}\right) .
\end{gathered}
$$

According to Green's theorem in $n$ dimensions, $\boldsymbol{\nabla} \cdot \mathbf{I}$ integrated over some volume $V$ equals $I \cdot v$ integrated over the area $S$ enclosing $V$, $v$ being the unit outward

${ }^{10}$ For example, Eq. (1.8) in Ref. 4 can be solved for a quantity $P_{i}$ knowing the metric tensor. With the aid of $(2.1)$ there, one finds thereby $\phi^{i}$ s $/ \phi$, from which $\phi$ is immediately calculated: $\phi$ equals $\left[\operatorname{det}\left(\phi^{i \alpha} / \phi\right)\right] .^{-1 /(n+1)} \phi_{i \alpha}$ is then obtained by inversion.
However, a much more direct method of obtaining $\phi_{i \alpha}$ from the However, a much more direct method
metric tensor can probably be found.

"However, the expression for a "probability current density" in curvilinear coordinates is given in Ref. $6, \mathrm{p}$. 40 . It equals $I \mathrm{~g}^{\frac{1}{4}}$, aside from a term of magnetic origin. No use was made of covariant and contravariant forms, and so the expression cited does not emphasize its invariant property. Nevertheless, it would lead to Eq. (24) when used to calculate $J$ for the current through a coordinate hypersurface.

12 For example, E. C. Kemble, The Fundamental Principles of Quantum Mechanics (Dover Publications, Inc., New York, 1958), p. 31 . drawn vector normal to $S .{ }^{13}$ Because of the probability interpretation of $p$ it then follows from (19) in the standard way that $J$, the probability current through $S$, is given by (21). It is, of course, a scalar

$$
J=\int_{S} \mathbf{I} \cdot \boldsymbol{v} d \sigma
$$

where $d \sigma$ is the area element of $S$.

The covariant and contravariant components of $\nabla$ along the coordinate curve $q^{j}$ are $\partial / \partial q^{j}$ and

$$
\sum_{j=1}^{n} \frac{g^{i j} \partial}{\partial q^{i}}
$$

respectively. ${ }^{13}$ The corresponding components of the vector $I$ are therefore seen from (20) to be

$$
I_{j}=\frac{\hbar}{2 i}\left(\psi^{*} \frac{\partial \psi}{\partial q^{j}}-\psi \frac{\partial \psi^{*}}{\partial q^{j}}\right) ; \quad I^{j}=\sum_{i=1}^{n} g^{i j} I_{i} .
$$

Inasmuch as the inner product $I \cdot \boldsymbol{v}$ is

$$
\sum_{j=1}^{n} I^{j_{\nu}}{ }_{j}\left(\text { and } \sum_{j=1}^{n} I_{j} \nu^{j}\right),,^{13}
$$

Eq. (21) is equivalent to

$$
J=\int_{S} \sum_{j=1}^{n} I^{j_{\nu_{j}}} d \sigma
$$

Although $S$ is a closed surface, it often happens that if one considers two bounded portions of $S$, joined by some connecting surface, and lets the two bounded regions increase in size indefinitely, the contributions of the current through the connecting portions ultimately becomes negligible. For example, this occurs when $\psi$ is square integrable over each of the first two infinite regions. If $\psi$ describes a wave packet and if we remove one of the two remaining surfaces to infinity, only the remaining surface contributes to $J$ in any finite time.

With these remarks as preliminary we consider the case that $S$ represents a single $q^{N}$-coordinate hypersurface, $S_{N}$, over which $\psi$ is square integrable. Only the $\nu_{N}$ term in (23) does not vanish ${ }^{14}$ and, as discussed in Appendix III, $\nu_{N} d \sigma$ equals

$$
g^{\frac{1}{2}} \prod_{i \neq N} d q^{i}
$$

${ }^{13} \mathrm{~A} . \mathrm{J}$. McConnell, Applications of the Absolute Differential Calcalus (Blackie and Son, Ltd., Glasgow, 1947).

14 If $v$ is the unit normal to $S_{N}$, then one can show $v_{j}=\delta_{i_{N}} / g^{N N}$, as follows: Let $\lambda$ be any vector which lies in $S_{N}$ but which is otherwise arbitrary. Since $S_{N}$ contains all coordinate curves but that of $q^{N}$ (each of them is at the intersection of $S_{N}$ with any $n-2$ other coordinate hypersurfaces) we can choose $\lambda$ so that only the $i$ th component $\lambda^{i}$ does not vanish $(i \neq N)$. $v \cdot \lambda$ equals $\Sigma v j \lambda$, i.e., $\nu_{i} \lambda^{i}$. Since $v$ is normal to $S_{N}, v \cdot \lambda$ vanishes and, therefore, so does $\nu_{i}(i \neq N)$. Since $\vee$ is a unit normal then $g^{N N_{\nu_{N} \nu_{N}}=1 \text {, i.e., }}$ $\nu_{N}=1 / g^{N N}$. $\nu^{N}$ equals $g^{N N_{\nu_{N}} \text {. }}$ 
The probability current $J$ through $S_{N}$ is therefore

$$
J=\int_{S_{N}} I^{N} g^{\frac{1}{2}} \prod_{i \neq N} d q^{i}
$$

where $I^{N}$ is given by (22).

\section{TRANSMISSION COEFFICIENT}

As in the usual Cartesian case,,$^{15}$ we take $J$ to be the probability current when the behavior of the wave packet is approximated by that of an infinite wave train (i.e., by an improper eigenfunction). The transmission coefficient $\kappa$ is defined to be the ratio of transmitted to incident probability current.

Let the wavefunction be given by (4), where

$$
\prod_{\mu \neq N} \psi_{\mu}
$$

is square integrable over the coordinate hypersurface $S_{N}$. We consider an open ${ }^{16}$ coordinate curve $q^{N}$ and note that for large values of $q^{N} \psi_{N}$ has the following asymptotic form..$^{17}$ (Set $N$ is now 1-dimensional.)

$$
\begin{aligned}
& \psi_{N} \underset{Q^{N} \rightarrow-\infty}{\longrightarrow} \exp \left(i \int \chi_{N^{\frac{3}{3}}} d q^{N}\right) \\
& +R \exp \left(-i \int \chi_{N^{\frac{3}{2}}} d q^{N}\right) / \chi_{N^{\frac{3}{3}} f^{N \frac{1}{2}},}
\end{aligned}
$$

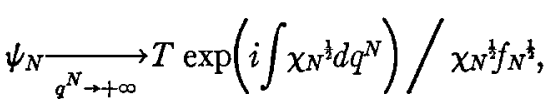

where

$$
\chi_{N}=\frac{2}{\hbar^{2}}\left(\sum_{\nu} \alpha_{\nu} \phi_{N \nu}-X_{N}\right)-\frac{1}{4}\left(\frac{d \ln f_{N}}{d q^{N}}\right)^{2}-\frac{1}{2} \frac{d^{2} \ln f_{N}}{d q^{N^{2}}},
$$

and where the region considered is one where $\chi_{N}$ is real. [For large $q^{N}$ in the cases we have examined, $f_{N}$ behaves as a power of $q^{N}$. Then, the second two terms in (26) vanish asymptotically.] Solution of the Schrödinger equation, subject to the boundary conditions (25), permits the determination of $T$. Using (24) and (I) one also finds

$$
J_{\text {trans }}=|T|^{2} \mathscr{G}, \quad J_{\text {inc }}=\mathscr{G}, \quad J_{\text {inc }}+J_{\text {refl }}=\left(1-|R|^{2}\right) \mathscr{I},
$$

\footnotetext{
${ }^{15}$ For example, L. D. Landau and E. M. Lifshitz, Quantum Mechanics (Addison-Wesley Publishing Company, Inc., Reading, Massachusetts, 1958), pp. 54 and 73.

${ }_{16}$ For example, a coordinate curve which extends to infinity. Under certain conditions (25) is also applicable over arcs of closed coordinate curves such as circles or ellipses.

${ }^{17}$ We have applied the method given in Jeffreys and Jeffreys, Methods of Mathematical Physics (Cambridge University Press, New York, 1962), p. 522, by first introducing a change of dependent variable $Y_{N}=\chi_{N} f_{N}{ }^{3}$ and then obtaining the asymptotic solution for $Y_{N}$ under the typical conditions of $\chi_{N}$ real. $\left[\exp \left( \pm \int i \chi_{N}{ }^{\frac{k}{3}} d q^{N}\right) / f_{N}^{3} \chi_{N}{ }^{\frac{1}{t}}\right.$ then describes an infinitely long wave train]. The $\chi$ here is $i x$ on p. 522.
}

where

$$
\mathfrak{g}=\hbar \int \phi \phi^{N 1} \prod_{\mu \neq N}\left(\left|\psi_{\mu}\right|^{2} f_{\mu}\right) \prod_{i \neq N} d q^{i}
$$

By definition, we have

$$
\kappa=J_{\text {trans }} / J_{\text {inc }}=|T|^{2} .
$$

Inasmuch as $\phi \phi^{N 1}$ and the remaining factors in (28) are independent of $q^{N}\left(\phi \phi^{N 1}\right.$ is the cofactor of $\phi_{N 1}$ in $\left.\phi\right)$, $g$ is independent of $q^{N}$, and so $J_{\text {ine }}$ and $J_{\text {trans }}$ are well defined. $\kappa$ depends only on the constants of the motion. Conservation of $J$ leads to $|T|^{2}$ being equal to $1-|R|^{2}$.

The quantity $\mathfrak{G}$ appears not only in (28) but elsewhere, as in Eq. (42) for $k=1$ (post). Normalization of wavefunctions of incident particles for which $\psi$ is not square integrable over $S_{N}$ is common in the literature, the normalization being to unit current density. In the present case of $\psi$ square integrable over $S_{N}$ a normalization to unit current would seem appropriate. We therefore set $\mathfrak{g}=1$.

\section{OPERATORS AND A VARIATIONAL PROPERTY FOR THE $\alpha_{p}$}

For possible applications in approximations, we obtain below the Hermitian operators for which the $\alpha_{p}$ are eigenvalues, and obtain thereby a stationary expression for the $\alpha_{\nu}$.

\section{a. Case of Discrete Spectrum for $\alpha_{\nu}$ 's}

Upon multiplying the $\mu$ th equation (17) by

$$
\phi^{\mu \lambda} \prod_{j \neq \mu}\left(\psi_{j} f_{j}\right) / \prod_{j \neq \mu} f_{j},
$$

summing over $\mu$, introducing (I), (II), and (18), noting that both

$$
\prod_{j \neq \mu} f_{j} \text { and } \phi \phi^{\mu 1}
$$

commute with $\partial / \partial q^{\mu i}$, and finally replacing $\lambda$ by $\nu$, one obtains

where

$$
H^{(\nu)} \psi=\alpha_{\nu} \psi,
$$

$$
H^{(\nu)}=\sum_{\mu=1}^{m}\left[-\frac{1}{2} \hbar^{2}(g)-\frac{\phi^{2}}{\phi^{\mu \nu}} \frac{\partial}{\partial q^{\mu_{i}}} g^{\frac{1}{1}} g^{\mu i \mu j} \frac{\partial}{\partial q^{\mu j}}+\phi^{\mu \nu} X_{\mu}\right] .
$$

When $\nu$ is $1, H^{(\nu)}$ is the Hamiltonian.

One may define an inner product (32), where $\psi_{A}$ and $\psi_{B}$ are any acceptable wavefunctions. Equation (33) follows from (30)

$$
\begin{gathered}
\left(\psi_{A}, \psi_{B}\right)=\int \psi_{A} * \psi_{B} g^{\frac{1}{3}} \prod_{i=1}^{n} d q^{i}, \\
\alpha_{\nu}=\left(\psi, H^{(\nu)} \psi\right) /(\psi, \psi) .
\end{gathered}
$$

The operator $H^{(\nu)}$ is Hermitian: Insamuch as $\phi^{\mu \nu} / \phi^{\mu 1}$ equals $\phi \phi^{\mu \nu} / \phi \phi^{\mu 1}$ and both $\phi \phi^{\mu \nu}$ and $\phi \phi^{\mu 1}$ are independent of the coordinates of set $\mu$, integration by parts shows that

$$
\left(\psi_{A}, H^{(\nu)} \psi_{B}\right)=\left(H^{(\nu)} \psi_{A}, \psi_{B}\right)
$$


Expression (33) has the desired variational property, it is stationary with respect to variations in $\psi$, for in the standard way one finds with the aid of (34):

$$
\begin{array}{r}
\delta d_{\nu}=\left\{\delta \psi,\left(H^{(v)} \psi-\left[\left(\psi, H^{(p)} \psi\right) /(\psi, \psi)\right] \psi\right)\right\} \\
+ \text { complex conjugate. }
\end{array}
$$

Upon using (30) and (32) one sees that $\delta \alpha_{\nu}$ vanishes. When one sets $\delta \alpha_{y}=0,(35)$ becomes an equation which may be solved for any variational parameters in $\psi$, remembering that these occur in the $m$ equations, $\nu=$ 1 to $m$.

We note that although $H^{(1)}$ is defined whether the Schrödinger equation is separable or not, the other $H^{(v)}$ in (31) are defined for the separable case (for only then are $\phi^{\mu v}$ and $X_{\mu}$ defined) or for the case that $\sum_{\mu} \phi^{\mu \nu} X_{\mu}$ vanishes.

\section{b. Continuous Spectrum Case, But All $\alpha_{v}$ 's Discrete If One $\alpha_{y}$ Prescribed}

We consider the behavior in a range of $q^{N}$ where the coordinate $q^{N}$ can be treated classically. This situation is the typical one at large values of $q^{N}$. Near this $q^{N}$, $\mathrm{Eq}$. (17) for $\mu=N$ may be replaced by the classical equation

$$
\frac{1}{2} p_{N}{ }^{2}+X_{N}=\sum_{\nu=1}^{m} \alpha_{\nu} \phi_{N \nu}
$$

where $p_{N}$ is the momentum conjugate to $q^{N}$ (cf. Appendix I of Ref. 5).

Let us prescribe the value of one $\alpha_{\nu}$, call it $\alpha_{k}$, and the value of $q^{N}$. We seek a stationary expression for the remaining $\alpha_{\nu}$ 's.

Let $M^{\mu \lambda}$ be the algebraic complement ${ }^{18}$ of $\phi_{\mu \lambda} \phi_{N k}-$ $\phi_{\mu k} \phi_{N \lambda}$ in the determinant, $\phi .(\lambda \neq k$.$) Then { }^{18}$

$$
M^{\mu \lambda}=\phi\left(\phi^{\mu \lambda} \phi^{N k}-\phi^{\mu k} \phi^{N \lambda}\right) .
$$

From (16) and (37) one deduces the property

$$
\sum_{\mu \neq N} M^{\mu \lambda} \phi_{\mu p}=\phi \phi^{N k} \delta_{p}{ }^{\lambda}-\phi \phi^{N \lambda} \delta_{p}{ }^{k} .
$$

Multiplying the $\mu$ th equation (17) by

$$
\left(\prod_{j \neq \mu, N} \psi_{j}\right) M^{\mu \lambda} / \phi \phi^{N k} \text {, }
$$

summing over all $\mu$ but $N$, using (I), (II), (18), and (38) and the fact that

$$
\prod_{j \neq \mu} f_{j} \text { and } \phi \phi^{\mu 1}
$$

commute with $\partial / \partial q^{\mu i}$, and finally replacing $\lambda$ by $\nu$, one obtains

where

$$
H_{N}{ }^{(\nu)} \psi^{\prime}=\alpha_{\nu} \psi^{\prime},
$$

$$
\psi^{\prime}=\prod_{\mu \neq N} \psi_{\mu},
$$

${ }^{18} \mathrm{M}$. Bocher, Introduction to Higher Algebra (The Macmillan Company, New York, 1907), p. 31. and where

$$
\begin{aligned}
& H_{N}^{(\nu)}=\sum_{\mu \neq N} \frac{M^{\mu \nu}}{\phi \phi^{N k}} \\
& \times\left(-\frac{2}{\hbar^{2} g^{\frac{1}{2}} \phi^{\mu 1}} \sum_{i, j} \frac{\partial}{\partial q^{\mu_{i}}} g^{\frac{1}{2} g^{\mu i \mu_{j}}} \frac{\partial}{\partial q^{\mu j}}+X_{\mu}\right)+\alpha_{k} \phi^{N \nu} / \phi^{N k} .
\end{aligned}
$$

Introduction of the abbreviation $(,)_{N k}$ defined in (42), where $\psi_{A}{ }^{\prime}$ and $\psi_{B}{ }^{\prime}$ are any two acceptable wavefunctions, multiplication of (39) by

$$
\psi^{\prime *} \phi \phi^{N \hbar} \prod_{\mu \neq N} f_{\mu}
$$

and integration over all $q^{j}$ but $q^{N}$ leads to (43).

$$
\begin{gathered}
\left(\psi_{A}{ }^{\prime}, \psi_{B}{ }^{\prime}\right)_{N k}=\hbar \int \psi_{A}{ }^{\prime *} \psi_{B}{ }^{\prime} \phi \phi^{N k} \prod_{\mu \neq N}\left(f_{\mu} \prod_{i \neq N} d q^{i}\right), \\
\alpha_{\nu}=\left(\psi^{\prime}, H_{N}{ }^{(\nu)} \psi^{\prime}\right)_{N k} /\left(\psi^{\prime}, \psi^{\prime}\right)_{N k .}
\end{gathered}
$$

One can also show ${ }^{19}$

$$
\left(\psi_{A}{ }^{\prime}, H_{N}{ }^{(\nu)} \psi_{B}{ }^{\prime}\right)_{N k}=\left(H_{N}{ }^{(\nu)} \psi_{A}{ }^{\prime}, \psi_{B}{ }^{\prime}\right)_{N k} .
$$

When the Stäckel coefficients are real, as they are in the systems discussed by Eisenhart and perhaps in all systems of interest in molecular dynamics ( $g^{r a}$ is real), one can show from (42) and (43) that the complex conjugate of $\alpha_{\nu}$ equals $\alpha_{\nu}$, i.e., $\alpha_{\nu}$ is real.

Because of (44) and (39), Expression (43) can then be shown to be stationary in $\alpha_{\nu}$ for variations in $\psi^{\prime}$.

We have refrained from calling $\left(\psi_{A}{ }^{\prime}, \psi_{B}{ }^{\prime}\right)_{N k}$ an inner product (and $H_{N}{ }^{(v)}$ Hermitian), for we did not show its positivity when $\psi_{A}^{\prime}$ equals $\psi_{B}{ }^{\prime}$. (It has the other properties ${ }^{20}$ of an inner product.) In some typical cases of interest this positivity is established in Appendix IV. However, regardless of the sign of $\left(\psi^{\prime}, \psi^{\prime}\right)_{N k}, \alpha_{\nu}$ is real and (43) is an extremum with respect to variations in $\psi^{\prime}$.

\section{APPENDIX 1. NOTE ON $\operatorname{det} W_{\mu \nu}$ NOT VANISHING IDENTICALLY}

Let $\rho_{\mu}$ denote

$$
\left(\psi_{\mu}\right)^{-1} \sum_{i, j=1}^{h_{\mu}} \frac{\partial}{\partial q^{\mu_{i}}} f^{\mu_{\mu} \mu_{j}} \frac{\partial \psi_{\mu}}{\partial q^{\mu_{j}}} .
$$

The $\rho_{\mu}$ are functions of the $\alpha_{\nu}$ and of the $q^{\mu_{i}}$. The coordinates $q^{\mu i}$ are treated as parameters for our purposes. If

$$
\operatorname{det}_{\mu, \nu=1}^{m} \rho_{\mu} / \partial \alpha_{\nu}
$$

vanished identically and if there were $m$ independent $\alpha_{y}$ 's $\left(\alpha_{1}\right.$ to $\left.\alpha_{m}\right)$ there would be a functional dependence

\footnotetext{
${ }^{18}$ Using (I) one sees that the factor multiplying the differential operator in the integrand is $-2 M^{\mu \nu} / h^{2} \phi^{\mu 1} f_{N}$. Both $M^{\mu \nu}$ and $\phi \phi^{\mu l}$ are independent of the coordinates in set $\mu$, since both are minors in $\phi$ not involving the $\mu$ th row. Equation (44) then follows upon by integration by parts.

${ }_{20} \mathrm{~B}$. Friedman, Principles and Teciniques of Applied Mathematics (John Wiley \& Sons, Inc., New York, 1956), p. 6, where the inner product is called a scalar product.
} 
between the $\rho_{\mu}$ 's, ${ }^{21}$ a dependence which could depend parametrically on the $q^{i}$. However, such a dependence would introduce one between the $q^{i}$ s. But these are independent variables, so $\operatorname{det} \partial \rho_{\mu} / \partial \alpha_{\nu} \neq 0$. On recalling the definition of $W_{\mu \nu}$ given in Eq. (7) one sees that $\operatorname{det} W_{\mu \nu} \neq 0$.

\section{APPENDIX II. ROTATING-VIBRATING DIATOMIC MOLECULE}

As a simple illustration we consider the diatomic molecule, the results for which are also easily obtained by standard methods.

If $r$ is the internuclear distance, $\theta$ and $\beta$ the angles defining the orientation of the molecular axis, and $M$ the reduced mass, let $\mu=1$ correspond to $r$ (vibration) and $\mu=2$ correspond to the set $(\theta, \beta)$ (rotation). Since $M^{-1}$ appears in all the $g^{r s} \mathrm{~s}$, we merely absorb it in the $k^{2}$, so $k^{2}$ becomes $2 M / \hbar^{2}$. From the Schrödinger equation for this system one finds $g^{11} \equiv g^{r r}=1, g^{22_{1}} \equiv g^{\theta \theta}=r^{-2}$, $g^{2222} \equiv g^{\beta \beta}=\left(r^{2} \sin ^{2} \theta\right)^{-1}, g^{21_{2} 2}=0$, whence $g^{\frac{1}{2}}=r^{2} \sin \theta$. From these results one finds ${ }^{22} \phi_{12}=r^{-2}, \phi_{21}=\sin \theta, \phi=\sin \theta$. Hence,

$$
\phi=\sin \theta=\left|\begin{array}{ll}
\phi_{11} & r^{-2} \\
\phi_{21} & \sin \theta
\end{array}\right| .
$$

Recalling that $\phi_{\mu \nu}$ is a function only of coordinates in set $\mu$, a solution to (A1) is seen to be: $\phi_{11}=1, \phi_{21}=0$.

Since $\phi / g^{\frac{1}{2}}$ equals $r^{-2}, \mathrm{Eq}$. (III) is satisfied and one may write $f_{1}=r^{2}, f_{2}=1$. Calculating the $f^{f^{\mu} \mu_{j}}$ from (18) and satisfying (II) by writing

$$
V(r, \theta, \beta)=X_{1}(r)+\left[X_{2}(\theta, \beta) / r^{2} \sin \theta\right],
$$

the separated equations are

$$
\begin{gathered}
\frac{1}{r^{2}} \frac{d}{d r} r^{2} \frac{d}{d r} \psi_{1}+\frac{2 M}{\hbar^{2}}\left(\alpha_{1}+\frac{\alpha_{2}}{r^{2}}-X_{1}\right) \psi_{1}=0 \\
\frac{1}{\sin \theta} \frac{\partial}{\partial \theta} \sin \theta \frac{\partial \psi_{2}}{\partial \theta}+\frac{1}{\sin ^{2} \theta} \frac{\partial^{2} \psi_{2}}{\partial \beta^{2}}+\frac{2 M}{\hbar^{2}}\left(\alpha_{2}-\frac{X_{2}}{\sin \theta}\right) \psi_{2}=0
\end{gathered}
$$

where

$$
\psi=\psi_{1}(r) \psi_{2}(\theta, \beta)
$$

and where the second term in (A2) is the only allowed potential energy term arising from external forces for which the equation is still separable. When $X_{2}$ vanishes, the constant of the motion $2 M \alpha_{2}$ becomes the square of the total angular momentum. To further separate (A4) into $\theta$ and $\beta$ equations, it would be necessary that $X_{2}(\theta, \beta)$ be the sum of $h_{1}(\theta) / \sin \theta$ and $h_{2}(\beta) / \sin \theta$, where $h_{1}(\theta)$ and $h_{2}(\beta)$ are arbitrary functions of $\theta$ and $\beta$, respectively.

\footnotetext{
2l E. B. Wilson, Advanced Calculus (Ginn and Company, Boston Massachusetts, 1912), p. 133.

22 From Eq. (II) one finds that $\phi^{11}=1$ and $\phi^{21}=1 / r^{2} \sin \theta$. Since $\phi \phi^{11}$ and $\phi \phi^{21}$ are the cofactors of $\phi_{11}$ and $\phi_{21}$ in the determinant $\phi$, they equal $\phi_{22}$ and $\phi_{12}$. One thus finds $\phi_{22} / \phi_{12}=r^{2} \sin \theta$. Since $\phi_{22}$ is a function of $(\theta, \beta)$ and $\phi_{12}$ is a function only of $r$ we can set them equal to sins and $r^{-2}$, respectively. Since $\phi \phi^{11}$ equals $\phi_{22}$, we then find $\phi=\sin \theta$.
}

\section{APPENDIX III. MAGNITUDE OF THE AREA ELEMENT}

The area element $d \sigma$ of $S_{N}$ is

$$
\left(g g^{N N}\right)^{\frac{1}{2}} \prod_{i \neq N} d q^{i}
$$

The proof is as follows: $d \sigma$ is the "volume element" in $S_{N}$ and so equals

$$
\left(\underset{i, j \neq \neq}{\operatorname{det}} g_{i j}\right)^{\frac{1}{2}} \prod_{i \neq N} d q^{i}
$$

[The volume element contains the determinant of the coefficients $g_{i j}$ appearing in the expression (2a) for the line element, $d s .{ }^{22}$ On $S_{N}$ all $g_{i j}$ occur in the line element except those for which $i$ or $j$ or both equal $N$.] Since the indicated determinant is also the cofactor of $g_{N N}$ in $g$, it equals $g g^{N N}$. The value cited for the area element follows.

A more instructive proof, perhaps, is obtained by noting that $d \sigma$ equals the $n$-dimensional volume element $d \tau$ divided by $\delta$, the perpendicular distance between $S_{N}$ and another $q^{N}$-coordinate hypersurface for which $q^{N}$ differs by $d q^{N}$. If $\delta$ is a vector normal to $S_{N}$ and having a length $\delta$ its contravariant component along $q^{N}$ must be equal to $d q^{N}$, by definition. Some manipulation then shows that $\delta=d q^{N} /\left(g^{N N}\right)^{\frac{1}{2}}$. The value cited for $d \sigma$ then follows. [The manipulation is somewhat similar to that ${ }^{13}$ involved in showing that a vector normal to $S^{N}$ has a contravariant component along $q^{N}$ equal to $\left(g^{N N}\right)^{\frac{1}{2}}$, if it has unit length.]

\section{APPENDIX IV. THE SIGN OF $\left(\psi^{\prime}, \psi^{\prime}\right)_{N k}$}

The positivity of $\left(\psi^{\prime}, \psi^{\prime}\right)_{N k}$ is easily established in at least a number of cases of physical interest:

(i) $k=1: \hbar\left(\psi^{\prime}, \psi^{\prime}\right)_{N 1}$ was shown earlier to be the incident probability current. Hence, $\left(\psi^{\prime}, \psi^{\prime}\right)_{N 1}$ is positive.

(ii) $k$ arbitrary: It has been shown elsewhere ${ }^{5}$ that $\left(\partial p_{N} / \partial \alpha_{k}\right)_{\lambda}$ equals

$$
\int\left|\psi^{\prime}\right|^{2} g^{\frac{1}{2}} \prod_{i \neq N} d q^{i} / p_{N} f_{N}\left(\psi^{\prime}, \psi^{\prime}\right)_{N k},
$$

where $\lambda$ is the totality of (discrete) quantum numbers characterizing $\psi^{\prime}$. The numerator is positive since

$$
g^{\frac{1}{2}} \prod_{i=1}^{n} d q^{i}
$$

is a volume element. $p_{N}$ and (without loss of generality) $f_{N}$ are also positive. When $p_{N}$ is a monotonic function of $\alpha_{k}$ at a given $\lambda$, and so has a constant sign, one can then choose the sign of $\alpha_{k}$ so that $\left(\partial p_{N} / \partial \alpha_{k}\right)_{\lambda}$ is positive.

In at least one typical choice of $\alpha_{k}$ of physical interest described elsewhere, ${ }^{5} p_{N}$ depends only on one $\alpha_{k}$; $\left(\partial p_{N} / \partial \alpha_{k}\right)_{\lambda}$ then equals $d p_{N} / d \alpha_{k}$ and, according to (36), equals $\phi_{N k} / p_{N}$. In that particular case $\phi_{N k}$ was of constant sign.

\footnotetext{
${ }^{23}$ Reference 7 (b) p. 42.
} 